\title{
Extragalactic Diffuse Interstellar Bands: A Universal Problem
}

\author{
M. A. Cordiner ${ }^{1,2}$ \\ ${ }^{1}$ Astrochemistry Laboratory and The Goddard Center for Astrobiology, NASA Goddard Space \\ Flight Center, Code 691, 8800 Greenbelt Road, Greenbelt, MD 20771, USA \\ email: martin.cordiner@nasa.gov \\ ${ }^{2}$ Department of Physics, The Catholic University of America Washington, DC 20064, USA
}

\begin{abstract}
The first extragalactic diffuse interstellar band (DIB) detections were of $\lambda 4430$ in the Large and Small Magellanic Clouds (LMC and SMC) in the 1960s and 70s. Driven mainly by the increased sensitivity afforded by $8-10 \mathrm{~m}$-class telescopes, the last 13 years have witnessed an explosion of DIB discoveries throughout the nearby and distant universe. This review focuses on the history of extragalactic DIB studies, including some of the important results that have come out of this field, and looks to the future for what can be learned about DIBs in external galaxies with the next generation of large telescopes. So far, DIBs have been observed in the Magellanic Clouds, starburst galaxies, DLAs, and nearby ( $\leqslant 30 \mathrm{Mpc}$ distant) spiral galaxies, and are found to be ubiquitous in the diffuse interstellar medium (ISM) of extragalactic environments wherever dust is present. Important results include the finding that DIB carriers are significantly more closely related to dust than to neutral hydrogen, and that the $\lambda 6283$ DIB tends to be anomalously weak in low-metallicity sightlines.
\end{abstract}

Keywords. ISM: lines and bands, galaxies: ISM, Local Group, quasars: absorption lines

\section{Introduction}

Previous studies of the DIB spectrum have been confined predominantly to sightlines within our own Galaxy. The Milky Way (MW), however, is just one among hundreds of billions of galaxies in the known universe. This therefore leads us to ask some questions of fundamental importance to DIB research, and to astrochemistry in general: Are DIBs present in other galaxies? If so, are their wavelengths and profiles similar to those found in the Milky Way? Do extragalactic DIBs show any correlations with the different interstellar conditions found in those galaxies?

The latter question is of particular importance in the context of the ongoing search for carrier assignments. A surprising outcome of MW DIB research has been the apparent consistency in DIB strengths, wavelengths and profiles across the range of physical and chemical conditions found in the MW. This consistency hinders correlation studies, which attempt to infer the composition of the carriers based on their correlations with interstellar variables. External galaxies span a wide range of different types, from spiral galaxies similar to our own to gas-poor ellipticals, dust-rich starburst galaxies, low-metallicity dwarves, damped Lyman-alpha systems and AGN. Such galaxies offer unique physical/chemical 'laboratories' in which to study the effect on the DIB spectrum of variations in factors such as the gas composition/metallicity, radiation field, temperature and dust properties. To this end, dedicated studies of diffuse interstellar bands in external galaxies have been underway since the late 1960s. 


\section{History of extragalactic DIB research}

Progress in extragalactic DIB research up to the turn of the century was reviewed by Snow (2002). The first reported extragalactic DIB observations were made by Walker (1963), who measured the strength of the $\lambda 4430$ DIB towards 10 LMC and 8 SMC stars. Dedicated studies of $\lambda 4430$ in the Magellanic Clouds (Hutchings 1966; Blades \& Madore 1979; Houziaux et al. 1980) have been unable to confirm any significant departure of the equivalent widths per unit reddening $\left(W / E_{B-V}\right)$ from the trend observed in the Milky Way. However, these observations have typically suffered from relatively low signal-tonoise ratios and small sample sizes, as well as the presence of overlapping stellar features. Observations of $\lambda 4430$ in the Magellanic Clouds are also hindered by an inability to accurately separate the foreground (MW) contribution from the extragalactic component due to the broad width of this DIB and the relatively low Doppler shift of the Magellanic Clouds. Nevertheless, an important result to come out of this early work was the fact that $\lambda 4430$ is relatively insensitive to the extreme variability of the $2175 \AA$ bump observed in the SMC, indicating that these features are not caused by the same carrier (Houziaux et al. 1985).

Pettini \& Dodorico (1986) reported the first detections of narrow DIBs $(\lambda \lambda 6376$ and $6379)$ towards two stars in the LMC using the ESO $3.6 \mathrm{~m}$ telescope. Soon after, Vladilo et al. (1987) and Vidal-Madjar et al. (1987) capitalized on the brightness of supernova 1987A to obtain very high signal-to-noise, high-resolution optical absorption spectra of the LMC ISM. By comparison with atomic interstellar absorption lines, these observations proved that DIBs in the LMC occur at approximately the same wavelengths as those in the Milky Way, and with similar profiles.

As an alternative to observing DIBs towards point sources, Heckman \& Lehnert (2000) used the integrated light from starburst galaxies to measure the equivalent width of extragalactic $\lambda 6283$ absorption. Reddenings were derived from stellar population modeling. Despite the unusual conditions in these galaxies (high star formation rates, intense UV radiation fields, high gas and dust densities and high temperatures), the DIB $W / E_{B-V}$ ratios all lie within the range observed in the Milky Way.

\section{Key discoveries in the era of $\mathbf{8 - 1 0}$ m-class telescopes}

Even for the relatively-nearby Small and Large Magellanic Clouds (at radial distances of 60 and $50 \mathrm{kpc}$, respectively), progress in extragalactic diffuse band studies was remarkably slow until the advent of the current generation of very large (8-10 m-class) telescopes in the late 1990s. Since then, the field of extragalactic DIB research has expanded rapidly as DIB surveys towards individual stars in Local Group galaxies, and towards distant background quasars have become feasible. Based on these recent observations, Figures 1 and 2 (for $\lambda 5780$ and $\lambda 6283$, respectively) show DIB strengths $(W)$ as a function of reddening $\left(E_{B-V}\right)$ for a variety of different galaxies. Some of the key results from this work are presented in the following sections.

\subsection{Large Magellanic Cloud}

The first high-resolution survey for DIBs towards LMC stars was presented by Ehrenfreund et al. (2002) and Cox et al. (2006), who detected DIBs towards 4 early-type LMC supergiants using the VLT UVES spectrograph. The LMC ISM is physically and chemically quite distinct from the Milky Way, with an average metallicity of around 0.5, a gas-to-dust ratio of four and a UV radiation field from a few to a few thousand times that of the Milky Way. Nevertheless, the LMC DIB spectrum was found to be quite similar to that of the Milky Way in terms of the bands present, their profiles and their 


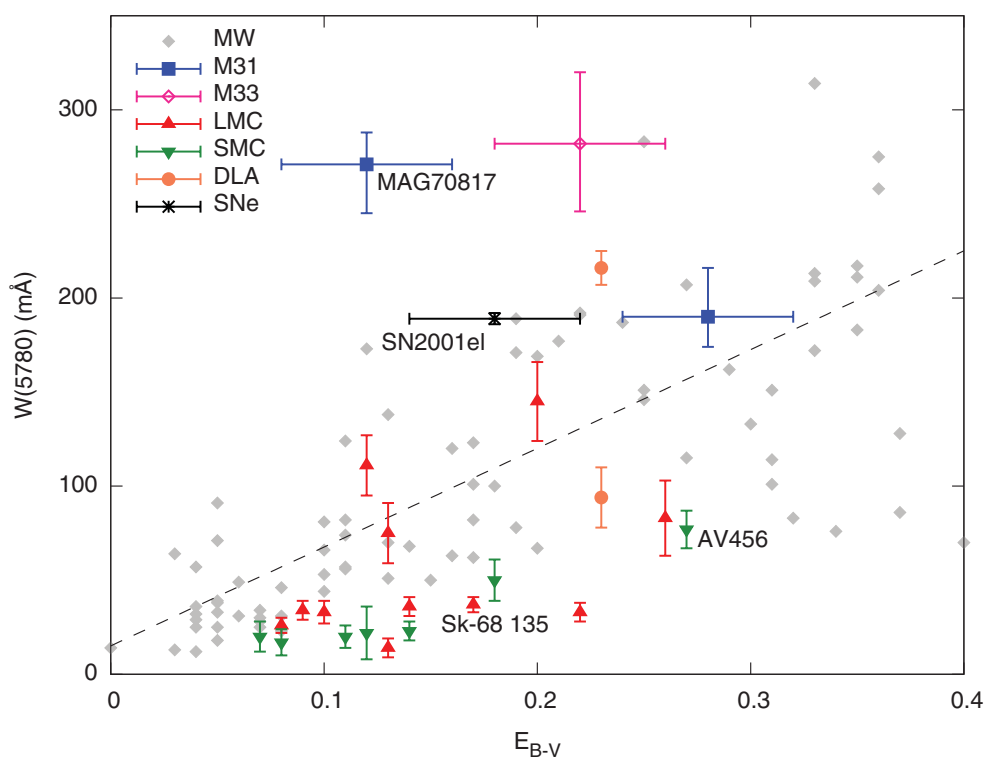

Figure 1. Observed $\lambda 5780$ DIB equivalent widths as a function of line-of-sight reddening for various galaxies, denoted by the different symbols. Dashed line shows a linear fit to the MW data of Friedman et al. (2011). LMC and SMC $E_{B-V}$ error bars have been omitted for clarity.

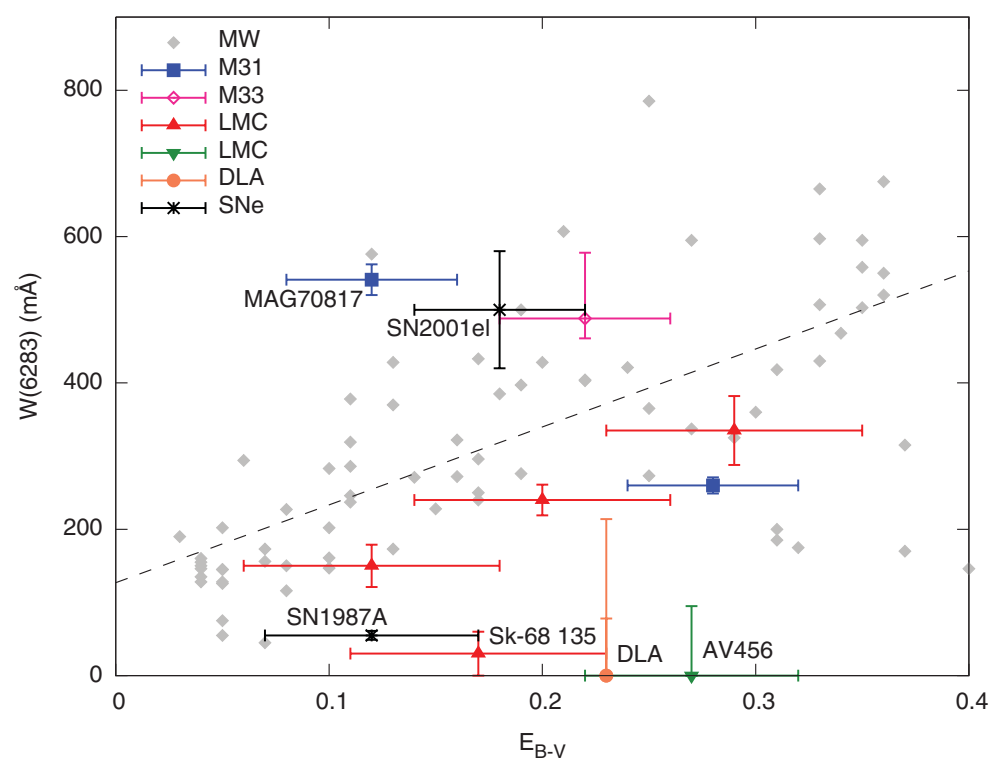

Figure 2. Same as Figure 1, for the $\lambda 6283$ DIB.

relative strengths. The $\lambda \lambda 5780$ and 5797 DIBs observed towards Sk -69223 are shown in Figure 3, compared to the (standard) MW star $\beta^{1}$ Sco and MAG 63885 in M 31.

Relative to $N(\mathrm{HI})$, the LMC DIBs are a factor of 5-10 times weaker than the Milky Way average, showing that the carriers are not closely related to atomic hydrogen gas. This result was confirmed for $\lambda \lambda 5780,5797$ and 6283 by Welty et al. (2006) in a larger sample of 13 LMC sightlines. The pattern of DIB strengths (as well as the Na I and K I column densities) in the LMC resembles that found in relatively strongly UV-irradiated Galactic 


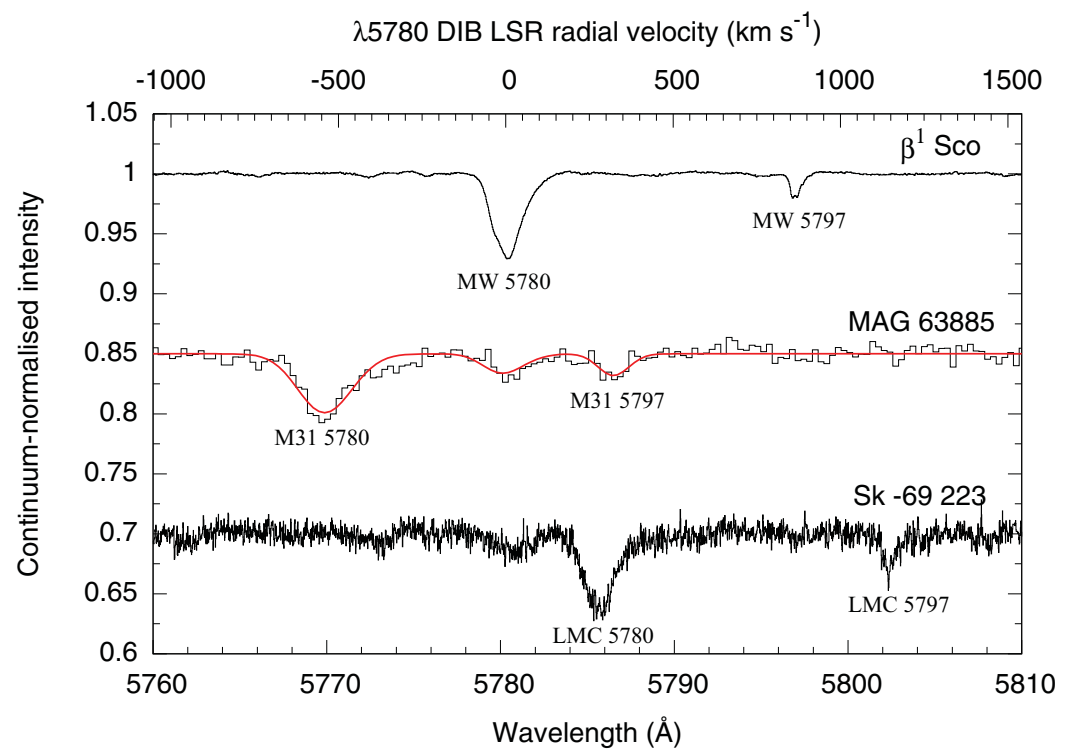

Figure 3. The $\lambda \lambda 5780$ and 5797 DIBs observed towards the Milky Way (MW) standard star $\beta^{1}$ Sco (top), the M 31 star MAG 63885 (middle), and the LMC star Sk -69 223 (bottom). Spectra have been normalised and offset vertically for display. The MAG 63885 data have a fitted profile overlaid in red, which includes M 31 and MW components (see Cordiner et al. 2008a for details). The DIBs are shifted by the Doppler effect for M31 and the LMC, as indicated by the horizontal scale at the top of the graph.

environments, although the DIB $W / E_{B-V}$ ratios are on average somewhat smaller than those typically found in the Galaxy.

As shown in Figure 2, the sightline towards $\mathrm{Sk}-68135$ is interesting due to its extremely small $W_{6283} / E_{B-V}$ ratio, which is about a factor of six less than the Galactic average (as measured, for example, by Yuan \& Liu 2012). The cause of such weak $\lambda 6283$ absorption is not established; this DIB is generally strong in the most strongly-irradiated Galactic diffuse clouds, but weakens in dense, dark, star-forming gas.

\subsection{Small Magellanic Cloud}

Six sightlines were observed in the Small Magellanic Cloud by Cox et al. (2006) and seven by Welty et al. (2006), with a combined total of eleven different sightlines. Again, Welty et al. (2006) identified a substantial weakening of $\lambda \lambda 5780,5797$ and 6283 . Relative to $N(\mathrm{HI})$, these DIBs are on average about a factor of twenty weaker than in the Milky Way, and relative to $E_{B-V}$, they are a factor of about 2 weaker (see Figures 1 and 2). The similar degree of weakening for both $\lambda \lambda 5780$ and 5797 in the SMC is at odds with the supposition that stronger UV-irradiation is the dominant factor responsible for the observed variations; in the Milky Way, stronger UV radiation fields tends to lead to weakened $\lambda 5797$ but enhanced $\lambda 5780$ and $\lambda 6283$ (e.g. Ehrenfreund \& Jenniskens 1995). Indeed, Welty et al. (2006) found no apparent relationship between the strengths of the DIBs and the intensity of the interstellar radiation field probed by molecular hydrogen. Thus, a possible explanation for the weakening of the DIBs in both Magellanic Clouds might involve the reduced metallicities, with the increased radiation field as a secondary 
effect. It may be that, as a result of a lack of a particular metal reagent $\dagger$, the carbon-rich molecules (or their chemical precursors), hypothesised as the most likely carriers of these DIBs, are produced in the ISM at a slower rate than the dust grains. This would result in reduced $W / E_{B-V}$ values as observed.

Similar to Sk -68135 in the LMC, AzV 456 in the SMC shows a relatively small $W_{6283} /$ $E_{B-V}$ ratio. Further studies of the chemical composition of the ISM in these sightlines could reveal important clues regarding the composition of the $\lambda 6283$ carrier.

\subsection{Andromeda Galaxy (M31)}

The first reported detection of diffuse interstellar bands in the Local Group beyond the Magellanic Clouds was by Cordiner et al. (2008a), who detected strong $\lambda \lambda 5780,5797$, 6203, 6283 and 6613 DIBs in sightlines towards two M 31 supergiant stars (MAG 63885 and MAG 70817) at radial velocities $\sim-550 \mathrm{~km} \mathrm{~s}^{-1}$, matching the Doppler shifts of the neutral (stellar + interstellar) sodium lines associated with M31. These detections were made using relatively low-resolution spectra obtained using Keck DEIMOS. The $\lambda \lambda 5780$ and 5797 DIBs observed towards MAG 63885 are shown in Figure 3, with a fitted profile overlaid. In such low-resolution spectra, only the broader DIBs (FWHM $\gtrsim 1 \AA$ ) have resolved profiles, and care must be taken to account for possible contributions from overlapping stellar and telluric features in the measurement of their strengths. Unfortunately, due to the $780 \mathrm{kpc}$ distance of M31, the brightest stars have $V$ magnitudes around 16, making high-resolution spectroscopy prohibitively time-consuming with current telescopes. Without adaptive optics, field crowding can also be problematic, making stellar spectral-typing (and hence, derivation of sightline reddenings) subject to potential uncertainty.

The sightlines examined by Cordiner et al. (2008a) are believed to be well-isolated however, and the surprising result that the DIBs towards MAG 70817 are very strong about a factor of three stronger than the Galactic average per unit $E_{B-V}$ (see Figures 1 and 2) - seems reasonably secure. Enhanced $\lambda 5780$ strengths were reported in 17 targets throughout the OB78 association in M31 by Cox \& Cordiner (2008), but these data suffer from considerable uncertainty due to to poor spectroscopic signal-to-noise.

Using the Gemini multi-object spectrograph (GMOS), Cordiner et al. (2011) made an optical survey of 34 sightlines in three regions of M 31, spanning a range of distances from the centre of that galaxy (shown in Figure 4). Diffuse interstellar bands were detected at M 31 radial velocities towards 11 OB supergiants. The relationships between DIB equivalent widths and reddening were found to be consistent with those observed in the local MW ISM, although a significantly large number of the observed sightlines have DIB $W / E_{B-V}$ values towards the upper end of the MW range. Thus, the formation and persistence of DIB carriers seems to be favoured in the relatively low-UV environment of M 31 that results from a low present-day star-formation rate. Understanding the root cause of these DIB strength enhancements could prove crucial in helping to identify the chemical composition of the carriers, and will require detailed measurements of the chemical and physical properties of the M31, which may be achievable using the next generation of ground and space-based telescope facilities.

\subsection{Triangulum Spiral (M 33)}

The Triangulum Spiral (M 33) is a smaller companion galaxy to M31, with a distance of about $880 \mathrm{kpc}$. Its basic properties were reviewed by Smith (2010) and Smith et al. (this

$\dagger$ In this context, a metal is any species heavier than $\mathrm{H}$ and $\mathrm{He}$. 

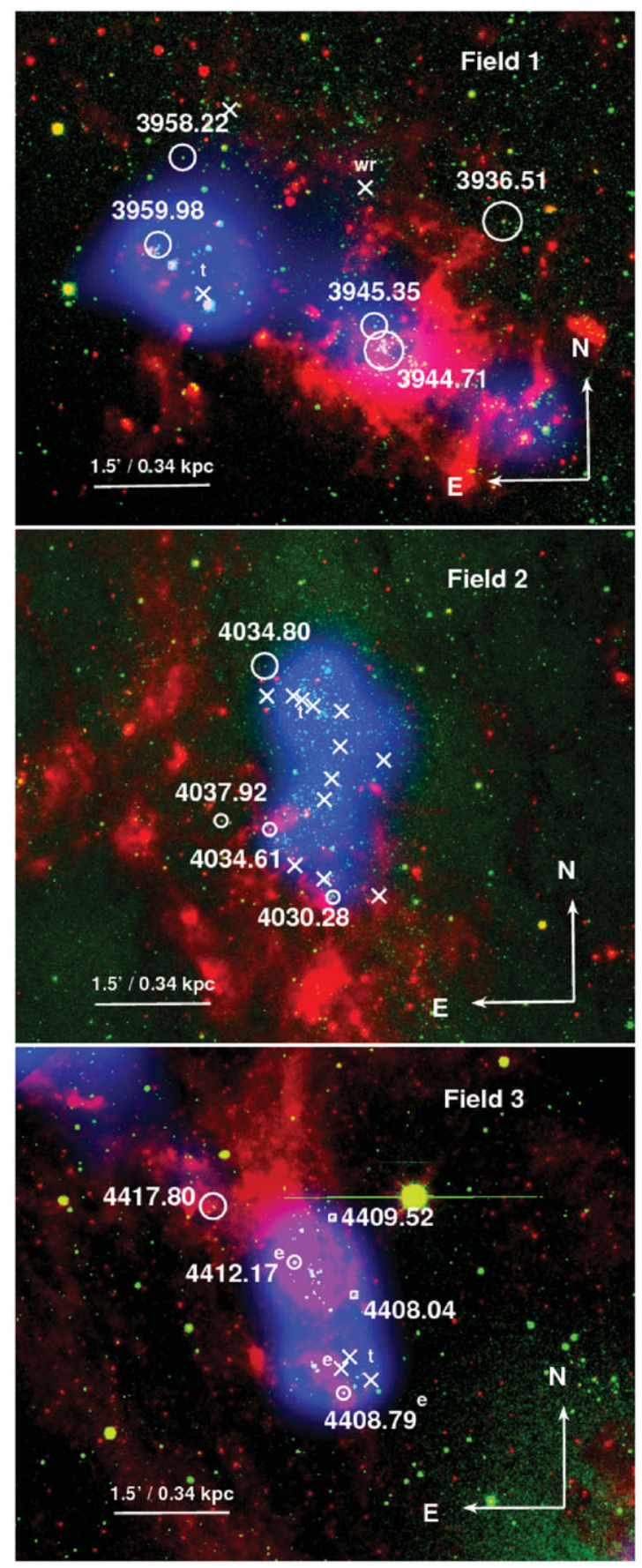

Figure 4. Three-colour composite maps of the M 31 fields observed by Cordiner et al. (2011). Green is optical ( $V$-band) imagery from Massey et al. (2006), red is the Spitzer $8 \mu \mathrm{m}$ (PAH emission), and blue is the NUV radiation field strength. Lines-of-sight with M 31 DIBs are marked with circles, the sizes of which represent the $W_{6283} / E_{B-V}$ value (relative to the M 31 mean): small, $10^{\prime \prime}$-circles denote weak; medium, $20^{\prime \prime}$-circles denote average and large, $30^{\prime \prime}$-circles denote strong DIBs (see Cordiner et al. 2011 for further details). Crosses indicate sightlines with no DIB detections, squares denote upper limits. Also marked are unreddened telluric standards (t), H II regions (e) and a Wolf-Rayet star observed in Field 1 (wr). 
volume), who presented details of the first survey for DIBs in this galaxy. The $\lambda 5780 \mathrm{DIB}$ was detected towards twenty stars, showing its carriers to be abundant throughout the ISM of M 33. Cordiner et al. (2008b) detected the $\lambda \lambda 5705,5780,5797,6196,6203,6269$, 6283 and 6613 DIBs at M 33 velocities (blueshiftd by about $140 \mathrm{~km} \mathrm{~s}^{-1}$ ), towards the supergiant star J013346.96+303642.8 in M 33. These DIBs were found to be remarkably strong (see Figures 1 and 2, for example), with $W / E_{B-V}$ values up to a factor of two greater than the Milky Way average. It is important to remember, however, that the M 33 sightlines presented so far were selected from a large sample of 43 stars based on their strong DIBs, leading to a biased sample. Further studies are required in order to determine the typical properties of the diffuse ISM in M 33.

Due to the low inclination angle of its disk, and its relatively small angular size (less than a degree), M 33 presents an ideal target for multi-object spectroscopy, which will facilitate the examination of variations in DIB properties on a galactic scale. Work is in progress (Smith \& Cordiner, in prep.), to obtain high-quality DIB spectra throughout the M33 disk, for comparison with known spatially-variable quantities such as the metallicity, PAH abundances, UV flux and star formation rate (see Magrini et al. 2007; Verley et al. 2009; Barnes et al. 2011). The increased signal-to-noise ratio offered by future large optical telescope facilities will be highly beneficial for this type of study.

\subsection{Supernovae}

The first reported detection of diffuse interstellar bands in the spectrum of a supernova was made by Rich (1987), who observed SN 1986G in Centaurus A using the CTIO $4 \mathrm{~m}$ telescope, and detected $\lambda \lambda 5780,5797,6283$ and 6613 at a redshift corresponding to the sodium D lines in the dust lane of the starburst galaxy. Their measured value for $W_{6613} / E_{B-V}$ is in line with the Milky Way average, but equivalent widths of the other DIBs were not given.

Several DIBs were detected by Vladilo et al. (1987) and Vidal-Madjar et al. (1987) in the line of sight towards SN 1987A in the LMC, providing the first reliable measurements of extragalactic DIB profiles. Some of the DIBs were found to be weaker than towards the Galactic sightline HD 183143, but the most noteworthy aspect of these results is the weakness of the $\lambda \lambda 6269$ and 6283 DIBs (Figure 2), which are significantly weaker (per unit reddening) than the Milky Way average.

Subsequent optical spectroscopy of supernovae throughout the nearby universe has revealed that DIBs are ubiquitous in (relatively-nearby) spiral galaxies, and that their strengths, wavelengths and profiles are generally quite similar to those observed in the Milky Way. DIBs were detected towards the Type Ia SN 2001el and Type II SN 2003hn in NGC1448 (20 Mpc distant) by Sollerman et al. (2005), and towards SN 2008D in NGC 2770 (30 Mpc-distant) by Thöne et al. (2008). Cox \& Patat (2008) observed extremely weak DIBs per unit reddening towards SN 2006X in M 100 (at $15 \mathrm{Mpc}$ ). These galaxies are all spirals, similar to the Milky Way in size and metallicity, so future studies would benefit from observations of supernovae in different kinds of galaxies. Supernova studies have provided DIB spectra of outstanding quality, but their usefulness is limited by the one-off nature of these events, which can tell us much about a single sightline, but little about the general properties of the galaxies in question.

\subsection{Damped Lyman-alpha systems}

So far this review has focussed on observations of targets that may be considered our immediate neighbours relative to the size of the observable universe. Observations of quasars provide the opportunity to probe interstellar absorption in much more distant 
galaxies, with the exciting prospect of measuring DIB properties as a function of the age of the universe. Damped Lyman-alpha systems (DLAs) comprise interstellar matter located in galaxies along the line of sight to a background quasar. The first detection of a diffuse interstellar band in a DLA was by Junkkarinen et al. (2004), who found $\lambda 4428$ at redshift $z=0.524$ (corresponding to a look-back time of about $5 \mathrm{Gyr}$ ), in the strong $\mathrm{H}$ I absorber towards AO 0235+164, with a $W / E_{B-V}$ value similar to MW. York et al. (2006) detected $\lambda \lambda 5780$ and 5705 in this DLA, again with strengths similar to MW, but $\lambda 6283$ was found to be anomalously weak, perhaps as a result of the low metallicity of the DLA. Ellison et al. (2008) observed an even weaker $\lambda 6283$ in a DLA at $z=0.16$ (see Figure 2 , in which the data points for these two DLAs overlap due to identical $E_{B-V}$ values).

Unfortunately, background quasars are faint, and DLAs as rich in dust as that seen towards AO $0235+164$ are rarely observed. Thus, despite dedicated surveys of multiple targets (e.g. Lawton et al. 2008; Ellison et al. 2008), DIBs have only been found in a total of three DLAs to-date the most recent detection was by Srianand et al. (2013).

\section{Conclusion}

Examination of the spectrum of diffuse interstellar bands in external galaxies has shown that the carriers of these unidentified features are abundant throughout the observable Universe, and were present in the ISM at high redshift as long ago as 5 Gyr. An important result from studies of the Magellanic Clouds and DLAs is that DIBs in galaxies across a range of dust-to-gas ratios correlate better with $E_{B-V}$ than $\mathrm{HI}$, proving that the composition of the DIB carriers is more closely related to (metal) dust constituents than to $\mathrm{HI}$.

The general consistency of diffuse band wavelengths, strength ratios and profiles across conditions of widely-varying metallicity, UV field, temperature and pressure show that the carriers are hardy and relatively easily produced - across a very broad range of physical and chemical conditions, interstellar chemistry tends to produce the same set of DIB carriers wherever dust is present.

Conversely, extragalactic observations have uncovered some of the most remarkable DIB spectra known, such as in the cases of Sk-68135 in the LMC, AzV 456 in the SMC, MAG 70817 in M 31, J013346.96+303642.8 in M33, SN 2006X in M 100 and in the DLAs. Detailed multi-wavelength followup studies of these sightlines are necessary in order to determine the cause of the anomalous DIB strengths, and have the potential to yield crucial new insights into the properties of the DIB carriers. Metallicity, for example, seems to be a likely candidate to explain the weakness of $\lambda 6283$ in some of these sightlines. This hypothesis can be confirmed with future surveys of external galaxies across a range of metallicities. A large sample of galaxies will be required in order to disentangle the effects of the various interstellar variables on the DIB strengths.

Our work on extragalactic DIB studies has only just begun. Many unexplored galaxies exist nearby within the Local group (at distances $<1 \mathrm{Mpc}$ ), accessible for detailed study with current instruments, although in many cases these have unfavourable Doppler shifts that preclude the separation of Milky Way DIBs from the target spectra. Beyond the Local Group, the number of viable targets increases rapidly, but individual sightline studies beyond $\sim 1 \mathrm{Mpc}$ are extremely challenging due to the difficulty of resolving individual stars and measuring weak DIBs in the noisy, necessarily low-resolution spectra of such faint targets. Future generations of more powerful optical facilities will therefore be instrumental in advancing the field of extragalactic DIB research. 


\section{References}

Barnes, K. L., van Zee, L., \& Skillman, E. 2011, ApJ, 743, 137

Blades, J. C. \& Madore, B. F. 1979, A\& A, 71, 359

Cordiner, M. A., Cox, N. L. J., Trundle, C., et al. 2008, A\&A, 480, L13

Cordiner, M. A., Smith, K. T., Cox, N. L. J., et al. 2008, A\&3A, 492, L5

Cordiner, M. A., Cox, N. L. J., Evans, C. J et al. 2011, ApJ, 726, 39

Cox, N. L. J., Cordiner, M. A., Cami, J., et al. 2006 A $\& A$, 447, 991

Cox, N. L. J., Cordiner, M. A., Ehrenfreund, P., et al. 2007, A\&SA, 470, 941

Cox, N. L. J. \& Patat, F. 2008, $A \& A$, 485, 9

Cox, N. L. J. \& Cordiner, M. A. 2008, IAUS, 251, 237

Ellison, S. L., York, B. A., Murphy, M. M. T., et al. 2008, MNRAS, 383, L30

Ehrenfreund, P. \& Jenniskens, P. 1995, in The Diffuse Interstellar Bands, ed. A. G. G. M. Tielens \& T. P. Snow (Dordrecht: Kluwer Academic Publishers), 202, 105

Ehrenfreund, P., Cami, J., Jiménez-Vicente, J., et al. 2002, ApJ, 576, L117

Friedman, S. D., York, D. G., McCall, B. J., et al. 2011, ApJ, 727, 33

Heckman, T. M. \& Lehnert, M. D. 2000, ApJ, 537, 690

Houziaux, L., Nandy, K., \& Morgan, D. H. 1980, A\&A, 84, 377

Houziaux, L., Nandy, K., \& Morgan, D. H. 1985, MNRAS, 215, 5

Hutchings, J. B. 1966, MNRAS, 131, 299

Junkkarinen, V. T., Cohen, R. D., Beaver, E. A., et al. 2004, ApJ, 614, 658

Lawton, B., Churchill, C. W., York, B., et al. 2008, AJ, 136, 994

Magrini, L., Vilchez, J. M., Mampaso, A., Corradi, R. L. M., \& Leisy, P. 2007, A $\& A, 470,865$

Massey, P., Olsen, K. A. G., \& Hodge, P. W. 2006, AJ, 131, 2478

Pettini, M. \& Dodorico, S. 1986, ApJ, 310, 700

Rich, R. M. 1987, AJ, 94, 651

Smith, K. T. 2010, PhD thesis, The University of Nottingham

Snow, T. P. 2002, in 17th IAP Colloq. on Gaseous Matter in Galaxies and Intergalactic Space, ed. R. Ferlet, M. Lemoine, J. M. Désert, \& B. Raban (Paris: Frontier Group), 63

Sollerman, J., Cox, N. L. J., Mattila, S., et al. 2005, A\&A, 429, 559

Srianand, R., Gupta, N., Rahmani, H., et al. 2013, MNRAS, 428, 2198

Thöne, C. C., Michalowski, M. J., \& Leloudas, G. 2008, ApJ, 698, 1307

Verley, S., Corbelli, E., Giovanardi, C., \& Hunt, L. K. 2009, A\&AA, 493, 453

Vidal-Madjar, A., Andreani, P., Cristiani, S., et al.1987, A\& A, 177, L17

Vladilo, G., Crivellari, L., Molaro, P., \& Beckman, J. E., 1987, A\&A, 182, L59

Walker, G. A. H. 1963, MNRAS, 125, 141

Welty, D. E., Federman, S. R., Gredel, R., Thorburn, J. A., \& Lambert, D. L. 2006, ApJS, 165, 138

Yuan, H. B. \& Liu, X. W. 2012, MNRAS, 425, 1763

York, B. A., Ellison, S. L., Lawton, B. et al. 2006, ApJ, 647, L29

\section{Discussion}

HenNing: I'm not sure about the result in M 31 that the DIB strengths are inverselydependent on the UV radiation field strength. Heckman \& Lehnert (2000) observed strong DIBs in starburst galaxies where the radiation fields can be thousands of times the Milky Way average.

Cordiner: We also know from observations of DIBs in the 30 Dor region of the LMC that diffuse band carriers can be abundant in such high-UV environments. It seems in general that the carriers are resistant to UV photolysis. A reason for the DIBs observed by Heckman \& Lehnert (2000) being strong despite the high radiation could be related to more rapid DIB carrier production in those environments as a result of the higher pressures in the ISM of starburst galaxies. 
Why is it necessary to resolve individual stars when performing extragalactic DIB surveys?

CORDINER: Spectra of individual stars are required in order to obtain the spectral types, which are needed to accurately derive line-of-sight reddenings.

Mulas: Can we gain any insight into DIB behaviour from observations of galaxies at different inclinations?

CORDINER: Yes, this would gives us the opportunity examine the different properties of DIBs in disk vs. halo gas. A hypothesis to explain the relatively strong DIBs observed in M 31 compared to the Milky Way is that in M31 we are probing lines of sight through the halo, whereas in the Milky Way we mainly probe the disk.

FOING: In order to better understand the conditions in external galaxies, we need more probes of physical variables such as the UV field and density, which can be obtained from UV spectroscopy.

WeLty: The line of sight towards Sk 143 (AzV 456) in the SMC is unusual because of its higher-than-average ratio of UV field to density. This could be a factor in the unusual DIB strengths in this sightline. 